\title{
Efeito da fibra de coprodutos agroindustriais e sua avaliação nutricional para coelhos
}

\author{
[Effect of agricultural by-products fiber and its nutritional evaluation for rabbits] \\ M. Retore, L.P. Silva, G.S.P. Toledo, I.G. Araújo \\ Departamento de Zootecnia - UFSM \\ Avenida Roraima, 1000 \\ 97105-900 - Santa Maria, RS
}

\begin{abstract}
RESUMO
Estudou-se a influência da fibra oriunda de diferentes fontes - feno de alfafa, polpa de cítrus e casca de soja - sobre o desempenho, coeficientes de digestibilidade, parâmetros sanguíneos e características da carne de coelhos submetidos às dietas experimentais. Os animais foram distribuídos em três tratamentos, que consistiram de: FA - ração-controle, com feno de alfafa; PC - substituição total do feno de alfafa por polpa de cítrus; e CS - substituição total do feno de alfafa por casca de soja. As diferentes frações da fibra advindas da polpa de cítrus e da casca de soja não influenciaram o desempenho dos animais aos 89 dias e mostram que esses ingredientes podem ser utilizados como substitutos do feno de alfafa na dieta de coelhos. A qualidade de fibra da polpa de cítrus reduziu os níveis séricos de triacilglicerol, colesterol e hemoglobina dos animais. A baixa quantidade de lignina em relação à celulose e à hemicelulose da casca de soja propiciou melhor coeficiente de digestibilidade dos nutrientes e maior maciez da carne, porém menor deposição proteica quando comparada com a dieta com polpa de cítrus.
\end{abstract}

Palavras-chave: coelho, polpa de cítrus, ligação catiônica, qualidade da fibra

\begin{abstract}
The influence of fiber - alfalfa hay, citrus pulp, and soybean hulls - in diets of rabbits was studied on performance, digestibility coefficients, blood parameters, and meat characteristics. The animals were allotted into three treatments that consisted in: control diet, with alfalfa hay; total substitution of alfalfa hay by citrus pulp; and total substitution of alfalfa hay by soybean hulls. The different fiber fractions from citrus pulp and soybean hulls did not affect animal performance at the $89^{\text {th }}$ day; thus, these ingredients can be used as substitute of alfalfa hay on diet of rabbits. Fiber quality of citrus pulp decreased blood triacylglycerol, cholesterol, and hemoglobin levels. Lower amount of lignin in relation to cellulose and hemicellulose of soybean hulls provided better nutrient digestibility coefficients and higher tenderness meat, however, lower protein deposition when compared with the citrus pulp diet.
\end{abstract}

Keywords: rabbit, citrus pulp, cation-exchange capacity, fiber quality

\section{INTRODUÇÃO}

É fato a essencialidade da manutenção de níveis adequados de fibra na dieta de coelhos para garantir o funcionamento normal do trato gastrintestinal do animal e para que o fenômeno da cecotrofia ocorra, o que se converterá em melhor desempenho. No entanto, faltam pesquisas para determinar com precisão a quantidade de fibra digestível necessária ao desenvolvimento adequado da população microbiana, a qual é responsável por parte da proteína digestível e por vitaminas consumidas pelos animais como produtos da fermentação (Santos et al., 2004).

A fibra da dieta exerce vários efeitos metabólicos e fisiológicos no organismo animal, sendo estes diferenciados conforme as frações que a

Recebido em 29 de junho de 2009

Aceito em 20 de setembro de 2010

E-mail: maretore@hotmail.com 
constituem: solúvel ou insolúvel. Tais efeitos podem ser decorrentes de alterações em funções fisiológicas, como a taxa de excreção endógena e a passagem do alimento pelo trato gastrintestinal (Refstie et al., 1999); alterações no bolo alimentar e digesta, tais como a capacidade de hidratação, o volume, o pH e a fermentabilidade (Van Soest, 1994; Annison e Choct, 1994); ou ainda, de alterações na população e na atividade da microbiota intestinal (Wenk, 2001). Portanto, não somente a quantidade da fração fibrosa mas também a qualidade, visualizada a partir do fracionamento dos constituintes da parede celular vegetal, devem ser consideradas no balanceamento de dietas para coelhos.

O feno de alfafa é considerado alimento fibroso de alta qualidade, pois possui boa digestibilidade e palatabilidade, sendo a fonte de fibra referência na alimentação de coelhos. Segundo Robinson (1999), esse ingrediente pode conter, com base na matéria seca (MS), 9 a $13 \%$ de cinzas, 2 a $3 \%$ de gordura, 15 a $25 \%$ de proteína bruta (PB), 20 a 35\% de carboidratos não estruturais, como açúcares, pectinas e amido, e 30 a $50 \%$ de carboidratos estruturais. No entanto, é o alimento de maior custo, assim sua substituição por alimentos mais acessíveis economicamente, de maior disponibilidade e oferta constante no mercado, como coprodutos resultantes do processamento de oleaginosas e frutas, é necessária para tornar a atividade mais rentável. Coprodutos, como a polpa de cítrus e a casca de soja, são produzidos em grande escala no País, apresentam boa digestibilidade e são ricos em fibra digestível, podendo participar de dietas para coelhos.

A polpa de cítrus é coproduto da indústria de suco de laranja. É composta pela casca, sementes e bagaço que, após o processo de extração do suco, ainda apresentam alto teor de umidade. Visando à comercialização desse ingrediente, a indústria promove a desidratação do material e consequente peletização (Giardini, 1994). Por possuir alta quantidade de carboidratos solúveis e fibra, é considerado alimento intermediário entre volumoso e concentrado (Fegeros et al., 1995). A polpa de cítrus, seca e peletizada, contém aproximadamente $6 \%$ de PB, $11 \%$ de fibra bruta e 70 a $75 \%$ de nutrientes digestíveis totais e, segundo Hall (2000), pode conter mais de $20 \%$ de sua MS composta por açúcares simples, sendo este teor variável de acordo com a espécie e o cultivar de cítrus que a originam. Segundo Watanabe et al. (2007), efeitos mais detalhados sobre fontes ricas em fibra solúvel (pectina), como a polpa de cítrus, foram pouco estudados e podem diferir daqueles da fibra insolúvel, por serem passíveis de fermentação microbiana, principalmente no ceco.

A casca de soja, coproduto da industrialização do grão, tem grande destaque no cenário nacional em virtude da alta produção de soja, sendo que representa de 7 a $8 \%$ do peso do grão (Restle et al., 2004). Esse produto destaca-se pela elevada oferta, pelos preços competitivos e pela composição bromatológica que se adequa à alimentação animal, pois, assim como a polpa de cítrus, possui elevada proporção de frações altamente digestíveis (celulose e hemicelulose), associada à baixa presença de lignina, um dos principais componentes que afeta a digestão da fibra (Oliveira, 2006). Segundo Anderson et al. (1988), a casca de soja inteira e livre de contaminação por farelo de soja possui $74 \%$ de fibra em detergente neutro (FDN) na MS, sendo $47 \%$ compostos de celulose e $23 \%$ de hemicelulose e, de acordo com Gentilini et al. (1996), possui 13\% de PB.

Este trabalho teve por objetivo estudar a influência da fibra oriunda de diferentes fontes feno de alfafa, polpa de cítrus e casca de soja sobre o desempenho, os coeficientes de digestibilidade aparente, os parâmetros sanguíneos e as características da carne de coelhos submetidos às dietas experimentais.

\section{MATERIAL E MÉTODOS}

Antes de formular as dietas experimentais e iniciar o ensaio biológico, os ingredientes testados - feno de alfafa, polpa de cítrus e casca de soja - foram analisados bromatologicamente, em duplicata, quanto às medidas de MS $\left(105^{\circ} \mathrm{C} / 12 \mathrm{~h}\right)$, cinzas $\left(550^{\circ} \mathrm{C} / 4 \mathrm{~h}\right)$, lipídios, pelo método de Bligh e Dyer (1959), e PB por meio da determinação de nitrogênio, pelo método de Kjeldahl ( $\mathrm{N} \times$ 6,25), de acordo com as técnicas descritas pela AOAC (Official ..., 1995).

O teor de fibra total (FT) foi determinado conforme o método enzímico-gravimétrico $\mathrm{n}^{\underline{0}}$ 985.29 e $\mathrm{n}^{\mathrm{o}} 991.42$ (Official ..., 1995). Os teores de FDN, fibra em detergente ácido (FDA), celulose e lignina foram determinados de acordo 
com a metodologia de Goering e Van Soest (1970). O teor de hemicelulose foi calculado como a diferença entre o teor de FDN e FDA. O conteúdo de fibra solúvel foi determinado subtraindo-se a FDN da FT. As enzimas utilizadas para determinação da FT foram a $\alpha$ amilase, protease e amiloglicosidase. As propriedades físico-químicas de capacidade de ligação ao cobre e capacidade de hidratação foram determinadas pelo método de McBurney et al. (1983) e McConnell et al. (1974), respectivamente.
Com base na análise laboratorial dos alimentos (Tab. 1), formularam-se três dietas experimentais (Tab. 2), seguindo-se as recomendações nutricionais para coelhos das principais tabelas de exigências nutricionais (Lebas, 1980; De Blas e Mateos, 1998), sendo que a PB e a energia digestível (ED) foram fixadas acima dos níveis recomendados, objetivando-se obter dietas com maior densidade nutricional, visto que elas seriam fornecidas na forma farelada aos animais.

Tabela 1. Composição bromatológica, fracionamento e propriedades físico-químicas da fibra dos ingredientes testados para coelhos em crescimento

\begin{tabular}{lccc}
\hline Parâmetro & Feno de alfafa & Polpa de cítrus & Casca de soja \\
\hline Matéria seca (\%) & 87,72 & 86,64 & 89,19 \\
Cinzas (\%) & 8,32 & 8,57 & 7,91 \\
Proteína bruta (\%) & 19,10 & 7,93 & 10,65 \\
Lipídios (\%) & 4,12 & 3,44 & 1,14 \\
Fibra total (\%) & 68,85 & 67,67 & 87,87 \\
Fibra em detergente neutro (\%) & 48,72 & 30,22 & 70,78 \\
Fibra em detergente ácido (\%) & 32,53 & 22,57 & 50,36 \\
Hemicelulose (\%) & 16,19 & 7,65 & 20,42 \\
Celulose (\%) & 27,85 & 21,08 & 47,78 \\
Lignina (\%) & 8,76 & 4,92 & 5,26 \\
Fibra solúvel (\%) & 20,13 & 37,45 & 17,09 \\
Capacidade de hidratação (g/g) & 5,80 & 4,80 & 5,30 \\
Capacidade de ligação ao cobre (mg/100g) & 555,70 & 944,49 & 713,17 \\
\hline
\end{tabular}

As dietas foram compostas majoritariamente por milho e farelo de soja, visando à substituição do feno de alfafa por coprodutos agroindustriais fontes de fibra: polpa de cítrus e casca de soja. Essas rações formaram os tratamentos: FA ração-controle, com feno de alfafa; PC substituição total do feno de alfafa por polpa de cítrus; e CS - substituição total do feno de alfafa por casca de soja.

O ensaio foi realizado no período de 24 de setembro a 11 de novembro de 2007. Foram utilizados 24 coelhos da raça Nova Zelândia Branco, machos e fêmeas, desmamados aos 33 dias de idade, com peso médio de $927 \pm 49 \mathrm{~g}$, distribuídos aleatoriamente entre os tratamentos (oito animais/tratamento) e alojados em gaiolas individuais, elevadas $70 \mathrm{~cm}$ do solo, com acesso livre à ração e água. O período de adaptação às dietas e instalações foi de sete dias e, após esse período, os animais foram submetidos às dietas experimentais dos 40 aos 89 dias de idade. A temperatura média máxima registrada no ensaio foi de $26^{\circ} \mathrm{C}$, e a média mínima de $15^{\circ} \mathrm{C}$, tomada diariamente às $8 \mathrm{~h}$ da manhã.
Durante a realização do experimento, foram feitas as pesagens das sobras de ração e dos animais para os cálculos de consumo de ração diário, ganho de peso diário e conversão alimentar. Para o ensaio de digestibilidade, foi feita a coleta total das fezes, 20 dias após o início do experimento, durante sete dias, a fim de se estimar os coeficientes de digestibilidade aparente da MS, matéria orgânica (MO), PB e FDN, de acordo com a fórmula de Schneider e Flatt (1975): Digestibilidade do nutriente = (nutriente ingerido $(\mathrm{g})$ - nutriente nas fezes $(\mathrm{g}) /$ nutriente ingerido $(\mathrm{g})) \times 100$.

No último dia experimental, após 12 horas de jejum, todos os animais foram pesados, e metade deles foi abatida, sendo coletado sangue para posterior quantificação de glicose, proteínas totais, hemoglobina, colesterol total e triacilglicerol. As análises foram realizadas com kits enzímico colorimétricos da marca Doles ${ }^{(\mathrm{R})}$ Goiânia, Brasil. 
Efeito da fibra de coprodutos...

Tabela 2. Composição percentual e química das rações experimentais para coelhos em crescimento (\% da matéria seca)

\begin{tabular}{|c|c|c|c|}
\hline Ingrediente & Feno de alfafa & Polpa de cítrus & Casca de soja \\
\hline Feno de alfafa & 15,00 & --- & --- \\
\hline Polpa de cítrus & --- & 18,02 & --- \\
\hline Casca de soja & --- & --- & 10,6 \\
\hline Milho & 52,46 & 44,18 & 51,43 \\
\hline Farelo de soja & 23,79 & 28,11 & 27,47 \\
\hline Casca de arroz & 5,00 & 6,52 & 5,00 \\
\hline Calcário calcítico & 0,38 & 0,21 & 1,28 \\
\hline Sal & 0,50 & 0,50 & 0,50 \\
\hline Mix min e vit* & 0,50 & 0,50 & 0,50 \\
\hline Fosfato bicálcico & 0,77 & 0,66 & 0,75 \\
\hline Óleo de soja & 1,60 & 1,30 & 2,45 \\
\hline Nutriente & \multicolumn{3}{|c|}{ Composição calculada com base na \%MS } \\
\hline ED (kcal/kg) & 3.000 & 3.000 & 3.000 \\
\hline FDN & 22,00 & 21,00 & 23,00 \\
\hline FDA & 13,70 & 14,80 & 15,00 \\
\hline Hemicelulose & 8,21 & 6,62 & 8,01 \\
\hline Celulose & 9,85 & 10,17 & 11,00 \\
\hline Lignina & 3,92 & 3,63 & 3,21 \\
\hline Fibra solúvel & 5,21 & 9,07 & 4,04 \\
\hline Fibra total & 27,21 & 30,07 & 27,04 \\
\hline $\mathrm{PB}$ & 18,00 & 18,00 & 18,00 \\
\hline $\mathrm{Ca}$ & 0,70 & 0,70 & 0,90 \\
\hline $\mathrm{P}$ & 0,45 & 0,45 & 0,45 \\
\hline
\end{tabular}

*Composição por kg do produto= vit A: 300.000UI; vit D: 50.000UI; vit E: 4.000mg; vit K3: 100mg; vit B1: 200mg; vit B2: 300mg; vit B6: $100 \mathrm{mg}$; vit B12: $1.000 \mathrm{mcg}$; ác. nicotínico: $1.500 \mathrm{mg}$; ác. pantotênico: 1.000mg; colina: $35.000 \mathrm{mg}$; Fe; 4.000mg; Cu: 600mg; Co: 100mg; Mn:, 4.300mg; Zn: 6.000mg; I: 32mg; Se: 8mg; metionina: $60.000 \mathrm{mg}$; promotor de crescimento: $1.500 \mathrm{mg}$; coccidiostático: $12.500 \mathrm{mg}$; antioxidante: $10.000 \mathrm{mg}$.

Para a obtenção da relação carne/osso, foram pesados, após a desossa, a carne e os ossos da perna traseira direita, aplicando-se os valores na fórmula de Rao et al. (1978): $\mathrm{RC} / \mathrm{O}=\mathrm{PCa} / \mathrm{PO}$, em que $\mathrm{RC} / \mathrm{O}$ é a relação carne/osso, $\mathrm{PCa}$ é o peso da carne crua $(\mathrm{g})$ e PO é o peso dos ossos (g). Após a desossa, utilizou-se a carne para medir as perdas de líquido por cozimento, por meio da fórmula: $\mathrm{PCo}=(\mathrm{PM}$ crua $-\mathrm{PM}$ cozida)/PM cru x 100, em que PCo é a perda de peso pelo cozimento (\%), PM crua é o peso da carne crua e PM cozida é o peso da carne cozida (Piles et al., 2000). As amostras foram colocadas em bandejas de alumínio e assadas em forno elétrico a $250^{\circ} \mathrm{C}$ por 15 minutos. A diferença em porcentagem entre $\mathrm{o}$ peso final e o inicial correspondeu à perda de líquido por cozimento.

Para a análise da maciez objetiva, as amostras destinadas à determinação de perdas por cozimento, depois de assadas e resfriadas à temperatura ambiente, foram submetidas ao teste da força de cisalhamento. Procedeu-se à remoção de quatro amostras de $1,27 \mathrm{~cm}$ de diâmetro, as quais foram cisalhadas ao meio no equipamento Warner-Bratzler Shear modelo 3000, conforme metodologia de Kerth et al. (1995).

O ensaio biológico foi realizado em delineamento inteiramente ao acaso, com três tratamentos e oito repetições, em que cada animal foi considerado uma unidade experimental. Os resultados obtidos foram submetidos a teste para detecção de valores anômalos (outlier) antes da análise de variância, sendo as médias comparadas pelo teste Duncan a $10 \%$ de significância. A análise estatística foi realizada utilizando-se o Programa Estatístico SPSS/1997, versão 8.0.

\section{RESULTADOS E DISCUSSÃO}

Para o período dos 40 aos 68 dias de idade (Tab. 3 ), houve diferença apenas para o ganho de peso diário, em que os animais dos tratamentos CS e FA apresentaram os valores mais altos. Não foi 
verificada diferença entre as fontes de fibra avaliadas sobre os demais parâmetros de desempenho. Considerando o período total, dos 40 aos 89 dias, os animais dos tratamentos FA e PC consumiram mais ração que os do tratamento $\mathrm{CS}$, provavelmente pelo melhor aproveitamento digestivo e metabólico (Tab. 4) da dieta com casca de soja, hipótese esta sustentada pela melhor conversão alimentar dos animais submetidos a este tratamento, seguida pela não significância de ganho de peso entre os tratamentos.

Tabela 3. Desempenho de coelhos dos 40 aos 68 dias e dos 40 aos 89 dias de idade

\begin{tabular}{lccc}
\hline Parâmetro & Feno de alfafa & Polpa de cítrus & Casca de soja \\
\hline Peso vivo 40 dias (g) & \multicolumn{3}{c}{$40-68$ dias de idade } \\
Consumo de ração (g/dia) & $67,00 \pm 67,21$ & $938 \pm 35$ & $943 \pm 46$ \\
Ganho de peso (g/dia) & $28,00 \mathrm{ab} \pm 2,20$ & $64,11 \pm 4,54$ & $62,73 \pm 3,81$ \\
Conversão alimentar & $2,39 \pm 0,09$ & $26,41 \mathrm{~b} \pm 2,03$ & $29,70 \mathrm{a} \pm 1,46$ \\
Peso vivo 68 dias (g) & $1685 \pm 126$ & $2,43 \pm 0,35$ & $2,11 \pm 0,17$ \\
\hline & \multicolumn{4}{c}{$1678 \pm 34$} \\
\hline Consumo de ração (g/dia) & $74,66 \mathrm{ab} \pm 3,98$ & $40-89$ dias de idade \\
Ganho de peso (g/dia) & $24,81 \pm 2,29$ & $78,62 \mathrm{a} \pm 6,17$ & $67,37 \mathrm{~b} \pm 5,70$ \\
Conversão alimentar & $3,00 \mathrm{ab} \pm 0,14$ & $25,45 \pm 2,02$ & $26,03 \pm 3,27$ \\
Peso vivo 89 dias (g) & $2,123 \pm 139$ & $3,09 \mathrm{~b} \pm 0,25$ & $2,59 \mathrm{a} \pm 0,33$ \\
\hline
\end{tabular}

Resultados expressos como média \pm desvio-padrão.

Médias seguidas de letras distintas, na linha, diferem entre si pelo teste Duncan $(\mathrm{P}<0,10)$.

De Blas e Villamide (1990) ressaltaram que a inclusão de altos níveis de polpa de cítrus (30$45 \%$ ) em dietas para coelhos não afetou a taxa de crescimento quando se forneceu fibra indigestível proveniente de outros alimentos nas dietas. Espíndola (1999) postulou que as rações balanceadas para não ruminantes são suficientemente ricas em polissacarídeos não amiláceos, como pectinas, betaglucanos e pentosanas, e podem prejudicar o processo digestivo à medida que aumentam a viscosidade do conteúdo intestinal. No entanto, o aumento da viscosidade está intimamente relacionado com o aumento da capacidade de hidratação da fibra, a qual foi semelhante entre as diferentes fontes testadas (Tab. 1). Desse modo, pode-se inferir que a polpa de cítrus não influenciou negativamente os eventos de digestão e absorção de nutrientes até a porcentagem testada $(18,0 \%$ de inclusão na ração).

As fontes testadas não causaram efeito significativo sobre o ganho de peso, mostrando que os coprodutos agroindustriais, polpa de cítrus e casca de soja, equivalem-se ao feno de alfafa, tradicionalmente utilizado nas rações para coelhos. Já a conversão alimentar foi melhor para os animais dos tratamentos FA e CS, o que é explicado pelos melhores coeficientes de digestibilidade dos nutrientes desses ingredientes (Tab. 4).

Tabela 4. Coeficientes de digestibilidade aparente da matéria seca (CDMS), matéria orgânica (CDMO), proteína bruta (CDPB) e fibra em detergente neutro (CDFDN) das dietas experimentais para coelhos em crescimento

\begin{tabular}{lccc}
\hline Parâmetro & Feno de alfafa & Polpa de cítrus & Casca de soja \\
\hline CDMS (\%) & $78,72 \mathrm{ab} \pm 4,85$ & $75,37 \mathrm{~b} \pm 5,46$ & $82,63 \mathrm{a} \pm 3,57$ \\
CDMO (\%) & $79,90 \mathrm{ab} \pm 4,64$ & $77,14 \mathrm{~b} \pm 5,16$ & $82,71 \mathrm{a} \pm 3,49$ \\
CDPB (\%) & $90,24 \mathrm{a} \pm 2,24$ & $86,83 \mathrm{~b} \pm 4,91$ & $92,45 \mathrm{a} \pm 2,45$ \\
CDFDN $(\%)$ & $32,65 \mathrm{~b} \pm 2,28$ & $35,43 \mathrm{ab} \pm 3,84$ & $39,58 \mathrm{a} \pm 4,81$
\end{tabular}

Resultados expressos como média \pm desvio-padrão.

Médias seguidas de letras distintas, na linha, diferem entre si pelo teste Duncan $(\mathrm{P}<0,10)$. 
O peso vivo dos animais aos 89 dias não diferiu entre os ingredientes testados. Arruda et al. (2003), ao trabalharem com diferentes fontes de fibra, feno de alfafa e casca de soja, também não observaram diferença entre as dietas para peso final dos animais.

Segundo De Blas e Wiseman (1998), o efeito da fonte de fibra sobre a digestibilidade da matéria seca e da matéria orgânica correlaciona-se diretamente com as características da parede celular do alimento fibroso. No entanto, os tratamentos FA e CS não diferiram quanto a estes coeficientes (Tab. 4), embora o tratamento CS tenha apresentado melhor coeficiente de digestibilidade da MS e MO. A casca de soja, por conter maior proporção de celulose e hemicelulose em relação à lignina (Tab. 1), deve ter proporcionado maiores tempo de retenção e disponibilidade dos nutrientes, assim como melhor degradação da fração fibrosa pela atividade microbiana cecal, contribuindo para melhor digestibilidade dessa dieta. Arruda et al. (2002) também constataram melhor aproveitamento dos nutrientes pelos coelhos alimentados com dietas que continham casca de soja, cujos valores médios de digestibilidade foram de $72,4 \%$ para MS e 73,1\% para MO, em comparação com as dietas que continham feno de alfafa (69,0 e 69,4\%, respectivamente).

Os melhores coeficientes de digestibilidade aparente da FDN para os tratamentos CS e PC são reflexos da menor lignificação, do maior teor de hemicelulose (casca de soja) e da maior quantidade de fibra solúvel (polpa de cítrus) (Tab. 1) dessas fontes e, possivelmente, do maior tempo de retenção para atividade fermentativa, aliado ao maior efeito antiperistáltico, contribuindo para melhor colonização e ação enzimática da microflora do ceco-cólon sobre a fração fibrosa (De Blas e Wiseman, 1998).

No presente trabalho, os coeficientes de digestibilidade da MS, MO e PB da dieta do tratamento PC foram mais baixos que os dos demais tratamentos pelo maior potencial de ligação catiônica (Tab. 1) dessa fonte, o que pode ter influenciado negativamente na digestibilidade dos nutrientes, quelando/indisponibilizando cofatores de enzimas digestivas e sais biliares.

Nas características de sangue (Tab. 5), os animais submetidos ao tratamento PC apresentaram níveis de triacilglicerol e colesterol mais baixos que os dos outros tratamentos, o que é indicativo indireto do efeito negativo/quelante da polpa de cítrus sobre os sais biliares. Diversos estudos têm demonstrado o efeito deste coproduto sobre a redução dos níveis séricos e hepáticos do colesterol total, devido ao aumento da quantidade de sais biliares e de gordura total excretados nas fezes (Mourão et al., 2005), pois a polpa de cítrus tem maior capacidade em "ligar" esses sais (Tab. 1), impedindo-os de serem reabsorvidos pelo epitélio intestinal.

Tabela 5. Níveis de triacilglicerol (TRG), colesterol (COL), hemoglobina (HEM), glicose (GLIC) e proteínas totais (PROT) do soro de coelhos abatidos aos 89 dias de idade

\begin{tabular}{lccc}
\hline Parâmetro & Feno de alfafa & Polpa de cítrus & Casca de soja \\
\hline TRG (mg/dL) & $53,54 \mathrm{a} \pm 8,09$ & $37,94 \mathrm{~b} \pm 4,33$ & $54,41 \mathrm{a} \pm 12,47$ \\
COL (mg/dL) & $113,21 \mathrm{~b} \pm 12,30$ & $66,23 \mathrm{c} \pm 0,93$ & $177,53 \mathrm{a} \pm 19,67$ \\
HEM (g/dL) & $7,82 \mathrm{~b} \pm 2,03$ & $4,55 \mathrm{c} \pm 0,85$ & $11,37 \mathrm{a} \pm 1,81$ \\
GLIC (mg/dL) & $136,10 \mathrm{a} \pm 9,49$ & $124,44 \mathrm{~b} \pm 0,80$ & $130,35 \mathrm{ab} \pm 4,75$ \\
PROT (g/dL) & $5,70 \mathrm{~b} \pm 0,38$ & $6,06 \mathrm{ab} \pm 0,24$ & $6,27 \mathrm{a} \pm 0,13$ \\
\hline
\end{tabular}

Resultados expressos como média \pm desvio-padrão.

Médias seguidas de letras distintas, na linha, diferem entre si pelo teste Duncan $(\mathrm{P}<0,10)$.

Diminuindo a circulação entero-hepática dos sais biliares no trato digestório, o organismo mobiliza colesterol para produzir novos sais biliares, essenciais ao metabolismo das gorduras (formação de micelas), consequentemente diminuindo a taxa de colesterol sérico do organismo (Jimenez-Vergara et al., 1999). Com menos sais biliares circulantes, a emulsificação das gorduras não será tão intensa, formando menos micelas, assim menos lipídio será absorvido pelo epitélio do duodeno e jejuno proximal, e, consequentemente, haverá menor nível de triacilglicerol no sangue.

A quantidade de hemoglobina foi afetada pela alta capacidade de ligação catiônica da fibra da polpa de cítrus (Tab. 1), a qual diminui a disponibilidade de muitos cátions bi e trivalentes, inclusive o ferro. O ferro, fazendo parte da molécula, é essencial para a produção de 
hemoglobina que, entre outras funções, é responsável pelo transporte de oxigênio para as células. Isso é verificado nos dados da Tab. 5, os quais mostram que o soro dos animais do tratamento PC apresentou níveis mais baixos de hemoglobina em comparação aos dos demais tratamentos. O resultado intermediário obtido no tratamento FA pode ter sido em função de compostos fenólicos existentes no feno de alfafa, os quais podem causar a lise das células (hemáceas).

As fibras solúveis do tipo pectina melhoram a tolerância à glicose, pois retardam a absorção e digestão dos carboidratos, mantendo nível mais baixo de glicose no sangue, observado no tratamento PC e CS (Stark e Madar, 1994). Esse fato também pode justificar os menores níveis de triacilglicerol (entrada mais lenta de glicose, menos formação de triacilglicerol no fígado) para o tratamento PC.

Os níveis de proteínas totais circulantes foram mais elevados no soro dos animais que consumiram as dietas dos tratamentos CS e PC. A casca de soja e a polpa de cítrus podem apresentar desbalanço de aminoácidos, sendo que a pior qualidade proteica dessas fontes, quando comparadas ao feno de alfafa, resulta em níveis mais elevados de proteína circulante.

Quanto à qualidade de carcaça (Tab. 6), nos animais alimentados com as dietas FA e PC, observou-se maior relação carne/osso, enquanto nos do tratamento $\mathrm{CS}$, menor valor, o que pode ser em função da menor deposição proteica. Este fato é subsidiado pelo alto nível de proteína circulante verificado nesse tratamento (Tab. 5).

Tabela 6. Relação carne/osso (RC/O), perdas por cozimento (PCo) e força de cisalhamento (FC) da carne de coelhos abatidos aos 89 dias de idade

\begin{tabular}{lccc}
\hline Parâmetro & Feno de alfafa & Polpa de cítrus & Casca de soja \\
\hline RC/O (\%) & $4,54 \mathrm{a} \pm 0,09$ & $4,74 \mathrm{a} \pm 0,20$ & $4,06 \mathrm{~b} \pm 0,23$ \\
PCo (\%) & $28,02 \pm 1,61$ & $26,39 \pm 2,76$ & $24,20 \pm 4,14$ \\
FC (kgf) & $1,90 \mathrm{~b} \pm 0,17$ & $1,97 \mathrm{~b} \pm 0,25$ & $1,53 \mathrm{a} \pm 0,29$ \\
\hline
\end{tabular}

Resultados expressos como média \pm desvio-padrão.

Médias seguidas de letras distintas, na linha, diferem entre si pelo teste Duncan $(\mathrm{P}<0,10)$.

Não foi observada diferença para perdas de líquido por cozimento da carne entre os ingredientes testados. Analisando-se o parâmetro de força de cisalhamento, este foi menor para a carne dos animais alimentados com a dieta CS. Essa maior maciez verificada na carne dos animais que consumiram a dieta com casca de soja talvez seja em função da melhor digestibilidade dos nutrientes, mobilizando mais água para o músculo. Ou ainda, esses animais podem ter acumulado maior quantidade de gordura na carcaça, fato justificado pelos maiores níveis de triacilglicerol e colesterol circulantes (Tab. 5).

\section{CONCLUSÕES}

As fontes de fibra testadas não afetaram o peso de abate dos animais. A dieta que continha casca de soja apresentou melhores coeficientes de digestibilidade dos nutrientes e elevou os níveis de triacilglicerol, colesterol e hemoglobina sanguíneos, bem como aumentou a maciez da carne. A inclusão de polpa de cítrus na dieta resultou em maior quantidade de carne na carcaça.

\section{AGRADECIMENTOS}

À Giovelli \& Companhia Ltda., de Guarani das Missões - RS, pela doação da casca de soja. Ao senhor João Adolfo Klohn, pela doação da polpa de cítrus.

\section{REFERÊNCIAS BIBLIOGRÁFICAS}

ANDERSON, S.J.; MERRILL, J.K.; McDONNELL, M.L. et al. Digestibility and utilization of mechanically processed soybean hulls by lambs and steers. J. Anim. Sci., v.66, p.2965-2975, 1988.

ANNISON, G.; CHOCT, M. Plant polysaccharides - their physiochemical properties and nutritional roles in monogastric animals. In: ALLTECH ANNUAL SYMPOSIUM, 10., 1994, Notthingham. Proceedings... Nottingham: University Press, 1994. p.51-56. 
ARRUDA, A.M.V.; LOPES, D.C.; FERREIRA, W.M. et al. Digestibilidade aparente dos nutrientes de rações contendo diferentes fontes de fibra e níveis de amido com coelhos em crescimento. Rev. Bras. Zootec., v.31, p.11661175, 2002.

ARRUDA, A.M.V.; PEREIRA, E.S.; MIZUBUTI, I.Y. et al. Importância da fibra na nutrição de coelhos. Semina: Cien. Agr., v.24, p.181-190, 2003.

BLIGH, E.C.; DYER, W.J. A rapid method of total lipid. Extraction and purification. Can. J. Biochem. Physiol., v.37, p.911-917, 1959.

DE BLAS, C.; MATEOS, G.G. Feed Formulation. In: DE BLAS, C.; WISEMAN, J. The nutrition of the rabbit. London: $\mathrm{Cab}$ Publishing, 1998. p.241-253.

DE BLAS, C.; VILLAMIDE, M.J. Nutritive value of beet and citrus pulps for rabbits. Anim. Feed Sci. Technol., v.31, p.239-246, 1990.

DE BLAS, C.; WISEMAN, J. The nutrition of the rabbit. London: Cab Publishing, 1998. p.241253.

ESPÍNDOLA, G.B. Valor fisiológico e nutricional da fibra vegetal para coelhos. In: SEMINÁRIO NACIONAL DE PESQUISA E TECNOLOGIA EM CUNICULTURA, 3., 1999, Jaboticabal. Anais... Jaboticabal, 1999.

FEGEROS, K.; ZERVAS, G.; STAMOULI, S. et al. Nutritive value of dried citrus pulp and its effects on milk yield and milk composition of lactating ewes. J. Dairy Sci., v.78, p.1116-1121, 1995.

GENTILINI, F.P.; LIMA, G.J.M.M.; RUTZ, F. et al. Valores de energia da casca de soja crua e tostada obtidos com suínos. In: CONGRESSO BRASILEIRO DE VETERINÁRIOS ESPECIALISTAS EM SUÍNOS. 8., 1997. Anais... Foz do Iguaçu-PR: ABRAVES, 1997. p.375-376.

GIARDINI, W.V. Polpa de cítrus. Rev. Criadores, n.64, supl., p.5, 1994.

GOERING, H.K.; VAN SOEST, P.J. Forage fiber analysis: apparatus, reagents, procedures and some applications. Washington, DC: USDA, 1970. (Agricultural Handbook, 379).

HALL, M.B. Neutral detergent-soluble carbohydrates nutritional relevance and analysis
.A laboratory manual. Gainesville: University of Florida, 2000. 42p.

JIMENEZ-VERGARA， M.; FURR, H.; FERNANDEZ, M.L. Pectin and psyllium decrease the susceptibility of LDL to oxidation in guinea pigs. J. Nutr. Biochem., v.10, p.118124, 1999.

KERTH, C.R.; MILLER, M.F.; RANSEY, C.B. Improvement of beef tenderness and quality with calcium chloride injection in beef loins 48 hours post mortem. J. Food Sci., v.73, p.750-756, 1995.

LEBAS, F. Les recherches sur l'alimentation du lapin: Evolution au cours dernieres années et perspectives d'avenir. In: WORLD RABBIT CONGRESS, 2., 1980, Paris. Proceedings... Paris: WRSA, 1980. v.2, p.1-17.

McBURNEY, M.I.; VAN SOEST, P.J.; CHASE, L.E. Cation exchange capacity and buffering capacity of neutral-detergent fibres. J. Sci. Food Agric., v.34, p.910-916, 1983.

McCONNELL, A.A.; EASTWOOD, M.A.; MITCHELL, W.D. Physical characteristics of vegetable foodstuffs that could influence bowel function. J. Sci. Food Agric., v.25, p.1457-1464, 1974.

MOURÃO, D.M.; SALES, N.S.; PINHEIROSANTANA, H.M. Biodisponibilidade de vitaminas lipossolúveis. Rev. Nutr., v.18, p.529539, 2005.

OFFICIAL methods of analysis. 16.ed. Washington: AOAC, 1995. 1018p.

OLIVEIRA, A.S. Casca de soja na alimentação de ruminantes. Cajati, SP: Serrana Nutrição Animal, 2006. (Bol. Técnico). Disponível em: <http://www.serrana.com.br/NutricaoAnimal/Bol etimTecnico/PDF/Fevereiro 2006.pdf> Acessado em: 23 jan. 2009.

PILES, M.; BLASCO, A.; PLA, M. The effect of selection for growth rate on carcass composition and meat characteristics of rabbit. Meat Sci., v.54, p.347-355, 2000.

RAO, D.R.; CHEN, C.P.; SUNKI, G.R. et al. Effect of weaning and slaughter ages on rabbit meat production. II. Carcass quality and composition. J. Anim. Sci., v.46, p.578-583, 1978. 
REFSTIE, S.; SVIHUS, B.; SHEARER, K.D. et al. Nutrient digestibility in atlantic salmon and broiler chickens related to viscosity and nonstarch polisaccharide content in different soybean products. Anim. Feed Sci. Techn., v.79, p.331-345, 1999.

RESTLE, J.; FATURI, C.; ALVES FILHO, D.C. et al. Substituição do grão de sorgo por casca de soja na dieta de novilhos terminados em confinamento. R. Bras. Zootec., v.33, p.10091015, 2004.

ROBINSON, P.H. Neutral detergent fiber (NDF) and its role in alfalfa analisys. In: CALIFORNIA ALFALFA SYMPOSIUM, 29., 1999, Fresno. Proceedings... Fresno: UC Cooperative Extension, 1999. p.60-67.

SANTOS, E.A.; LUI, J.F.; SCAPINELLO, C. Efeito dos níveis de fibra em detergente ácido sobre os coeficientes de digestibilidade das dietas e desempenho de coelhos em crescimento. Acta Sc. Anim. Sci., v.26, p.79-86, 2004.
SCHNEIDER, B.H.; FLATT, W.P. The evaluation of feeds through digestibility experiments. Athens: University of Georgia, 1975. 423p.

STARK, A.; MADAR, Z. Dietary fiber. In: GOLDBERG, I. (Ed.). Functional foods. New York: Chapman and Hall, 1994. p.183-201.

VAN SOEST, P.J. Carbohydrates. In: Nutritional ecology of the ruminant. Ithaca: Cornell University, 1994. Cap.11, p.156-176.

WATANABE, P.H.; THOMAZ, M.C.; RUIZ, U.S. et al. Avaliação biológica da polpa cítrica para suínos em terminação. In: REUNIÃO ANUAL DA SOCIEDADE BRASILEIRA DE ZOOTECNIA, 44., 2007, Jaboticabal. Anais... Jaboticabal, 2007. CD-ROM.

WENK, C. The role of dietary fiber in the digestive physiology of the pig. Anim. Feed Sci. Tech., v.90, p.21-33, 2001. 